\title{
The SOM Robustness Capacity for Phonemes Recognition in Adverse Environment
}

\author{
Mohamed Salah Salhi \\ National school for Engineers \\ LP 37, the Belvédère, 1002 \\ Tunis,Tunisia
}

\author{
Najet Arous \\ National school for Engineers \\ LP 37, the Belvédère, 1002 \\ Tunis,Tunisia
}

\author{
Noureddine Ellouze \\ National school for Engineers \\ LP 37, the Belvédère, 1002 \\ Tunis,Tunisia
}

\begin{abstract}
In this paper, we describe the formatting guidelines for IJCA Journal Submission. The SOM, for kohonen Self Organizing Map, has proven to be a classifier of high caliber in the field of speech recognition signals breasts. Thus, several versions and enhancements were applied on this tool such as recurrent SOM 'RSOM', the growing recurrent SOM 'GRSOM' and the growing hierarchical SOM GHSOM, to consider the integration of sound variability. This paper aims to detect the ability of the SOM robustness in terms of phonemes recognition for continuous speech in a noisy environment. This idea represents, in fact, the real case for speech recognition.
\end{abstract}

\section{General Terms}

This paper is generally classified in the profile of Pattern Recognition and signal processing.

\section{Keywords}

Noisy environment; phonemes recognition; Self organizing map SOM; SOM robustness.

\section{INTRODUCTION}

The field of phonemes recognition is a very important area. It participated in the simplification of voice recognition, speaker identification, man-machine speech communication... Several classifiers were used for phonemes recognition since 1982 such as: HMM, SVM and neural networks. The majority of these methods are based on a supervised learning algorithm. In the case of processing a large data dictionary, an unsupervised learning algorithm is most appropriate. In that, the SOM model is proved as a powerful tool for phonemes recognition.
In speech recognition, this model has been known since the 80 's by the Finn T. Kohonen.

N. Arous used the SOM in speech recognition in the 2000s by the hybridization of GA and SOM. For this, as Kohonen, she uses three central windows of the phoneme.

C. Jlassi in (2006-2010) presents the hierarchical SOM 'HSOM' and the growing hierarchical SOM 'GHSOM', she also has used three central windows of the phoneme. Our contribution is:

-The implementation of the SOM on all windows of the phoneme.

\footnotetext{
-Improving the robustness of the SOM in a noisy environment.
}

After this introduction, our paper will deal with the SOM principle and the essentially comparative study for some methods in the second section. In section three we will focus on the examination of SOM robustness in the noise presence.

However, the experimental results and their argumentations are integrated respectively below each section. Finally, the section four is dedicated to the conclusion.

\section{SOM OPERATION}

Moving to the SOM principle in phonemic recognition, this model is proposed by T. Kohonen in 82. It was inspired by the biology of the human brain. It may be two dimensional or string. It uses the following rules:

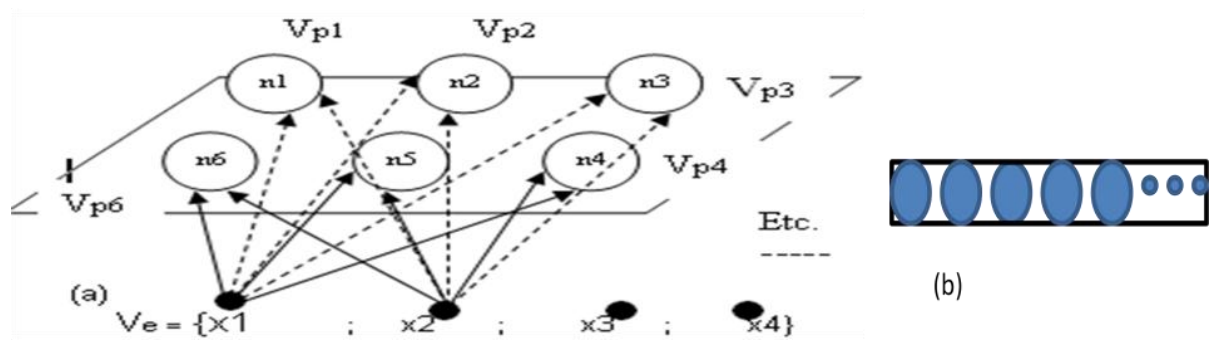

Fig.1 Representation of the SOM map; (a) in dimension 2 and (b) in string

The SOM map is based on the following Rules [1]:

-Each component of the input vector will be sent to each neuron of the SOM map.
-At each learning iteration end, the weight vectors, called prototypes and initialized linearly or randomly in the start, will have each one the same components of the input vector. 
-The quantization error associated to a neuron (i) is calculated by the Euclidean distance as follows:

$$
E_{i}=\left\|x(t)-w_{i}\right\|
$$

The winner neuron (v) also called BMU, is the unit that minimizes the quantization error from which we have:

$$
E_{v}=\min E_{i} \quad ; i \in N
$$

The learning rule updates the neural weights located in the vicinity of the active neuron by bringing them close to the input vector:

$$
\Delta w_{i}=\gamma \cdot h_{i v}\left(x(t)-w_{i}\right)
$$

$\gamma$ is a learning report and hiv is a neighborhood function, which decreases by the distance between units (i) and (v) on the SOM map.

However, the SOM disadvantage results in its convergence to a local optimum and the algorithm is not initially adapted to process the temporal dimensions of speech signal.

\section{SOM EXPERIMENTATION FOR HOLY SPEECH RECOGNITION}

Our work is experienced on the TIMIT database by considering its wide dissemination in the international community makes an objective assessment and sharing of desired performance of developed recognition systems.

Applied to phoneme recognition, our program performs the segmentation of sentences into $10 \mathrm{~ms}$ sound atoms.

We have used the total energy of each phoneme for the data parameterization.

The adopted approach for phonemic recognition is abstracted as follow:

-Reading and segmentation of the TIMIT database sentences. -Convert sound atoms in acoustic vectors consisting of coefficients MFCCs.

-Sequential presentation of these vectors into the SOM map.

-The MFCCs are calculated on all the phonemes windows where the energy is considered maximum.

The table and figure below show the results of the phonemes recognition in their classes by SOM.

Table I. Phonemic recognition rate of TIMIT database by SOM

\begin{tabular}{|c|c|c|c|c|}
\hline \multirow{2}{*}{$\begin{array}{c}\text { Phonemes } \\
\text { classes }\end{array}$} & \multicolumn{2}{|c|}{ Training } & \multicolumn{2}{c|}{ Nest } \\
\cline { 2 - 5 } simples & Rates & $\begin{array}{l}\text { Nbr of } \\
\text { simples }\end{array}$ & Rates \\
\hline Vowels & 4432 & 74.95 & 1339 & 52.89 \\
\hline Semi-vowels & 2717 & 86.57 & 844 & 70.99 \\
\hline Nasales & 1109 & 94.32 & 332 & 64.45 \\
\hline Fricatives & 1729 & 82.95 & 528 & 59.43 \\
\hline Stops & 3946 & 75.95 & 1122 & 48.86 \\
\hline Others & 239 & 75.95 & 75 & 52.75 \\
\hline Affriquates & 172 & 90.03 & 42 & 82.29 \\
\hline Overall rate & $\mathbf{1 4 3 4 4}$ & $\mathbf{8 2 . 9 6}$ & $\mathbf{4 2 8 2}$ & $\mathbf{6 1 . 6 6}$ \\
\hline
\end{tabular}




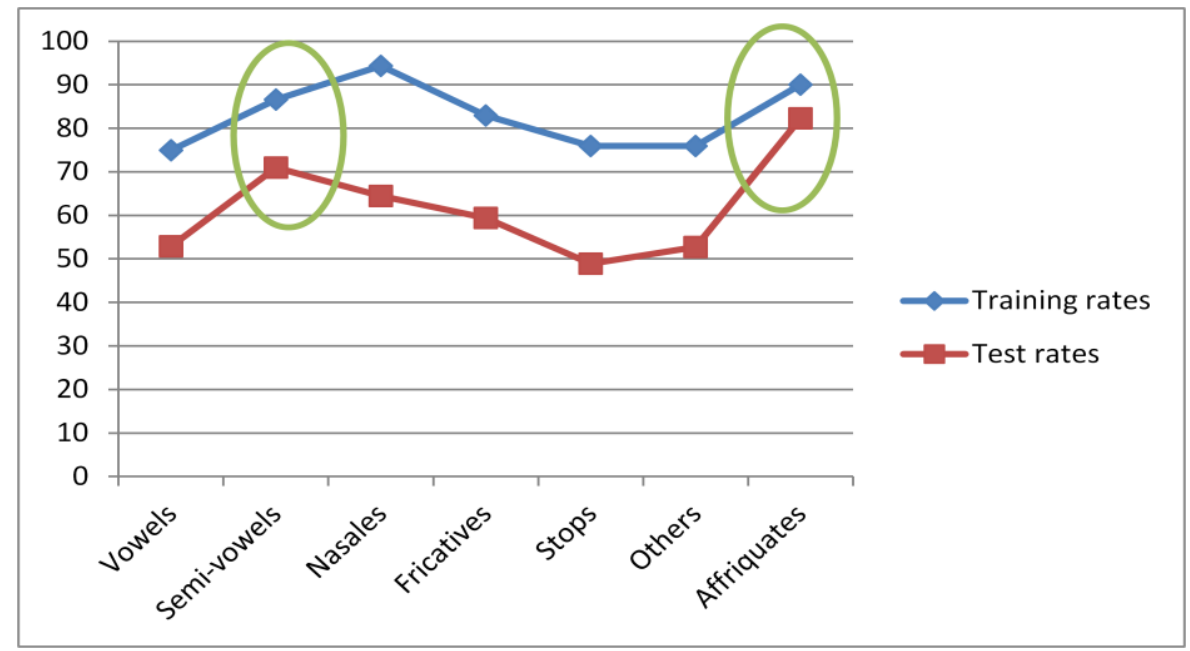

Fig.2 Representation of Training and test rates for all classes of TIMIT database

We note that for certain classes such as semi-vowels and affricates, the generalization (test) rates are closed to self consistency (training) ones due to their transient state.

A comparative study on the vowels basis shows the performance of SOM taking account of all the phoneme windows compared to SOM on three central windows. The obtained results confirm that they are better than those obtained by other models using three central windows.

Table II. Comparative recognition rates over models on vowels of TIMIT database by SOM

\begin{tabular}{|c|c|c|}
\hline Recognition model & Phoneme Support & Global generalization rates \\
\hline SOM N.Arous (2003) & 3 central windows & $\begin{array}{l}81 \% \text { for } \\
\text { training }\end{array}$ \\
\hline GHSOM C.Jlassi (2010) & 3 central windows & $\mathbf{5 6 \%}$ \\
\hline SOM M.S.Salhi (2011) & All windows & $\begin{array}{l}\mathbf{7 5 \%} \text { for } \\
\text { training }\end{array}$ \\
\hline
\end{tabular}

We have noted that In light of using all the phoneme windows, we have made a gain in generalization rate of $6 \%$ to $7 \%$.

These results are interpreted as follows:

-The SOM is a powerful tool in the representation of static data.

-The effectiveness in phoneme recognition, is strongly linked to the learning quality (number of windows, extended, overlap, etc..).

The study of the SOM robustness in the noise presence is another contribution that will be the subject of the next section.

\section{The SOM MODEL WITH NOISE REDUCTION IN PHONEMIC RECOGNITION}

Several noise reduction techniques have been known to use on the speech signal. Some techniques are classified in the spectral attenuation. Others belong to the atomic decomposition. The others are supported to statistical and hearing modeling [2-17].

In our strategy of speech recognition, we have adopted an atomic decomposition technique, which is the technique of wavelet packet denoising. 


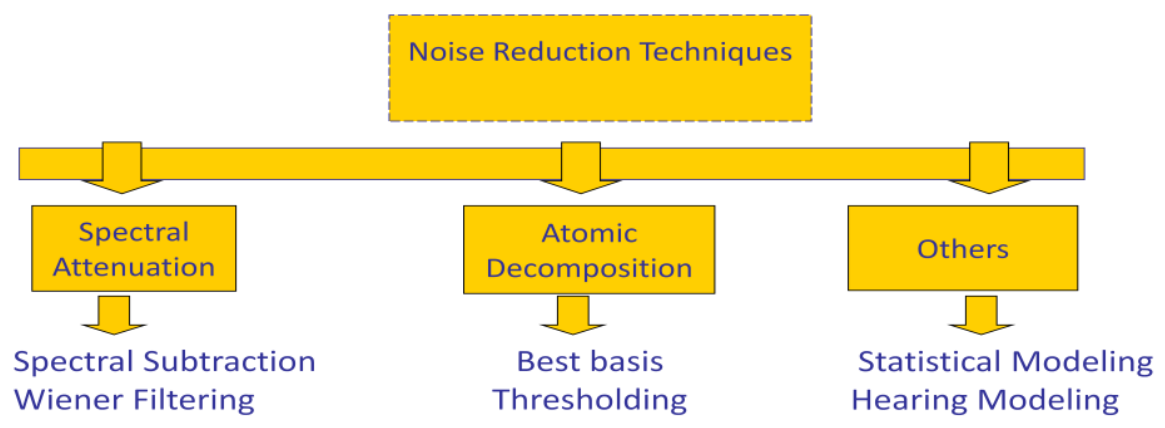

Fig.3 Representation of noise reduction techniques

\section{DENOISING BY WAVELET PACKET ANALYSIS AND ADAPTED THRESHOLDING}

The method of wavelet packet decomposition that we used is

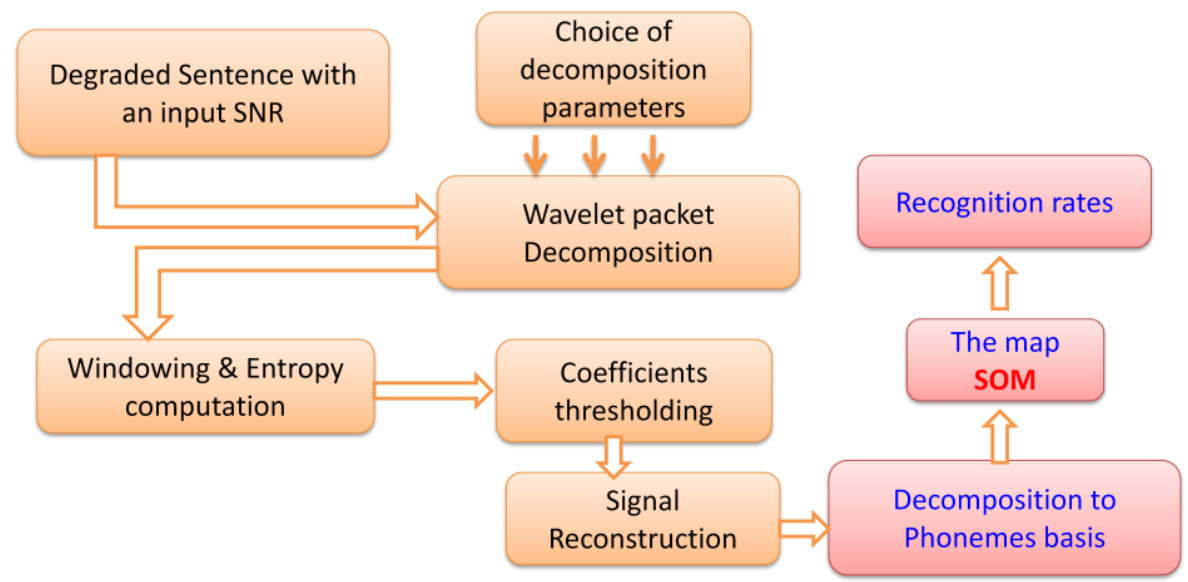

Fig.4 Diagram of adopted recognition algorithm

Contrary to the dyadic wavelet transform (DWT), wavelet packets decompose the signal by low-pass filters and high pass filters [18].

The following figures illustrate well this idea.

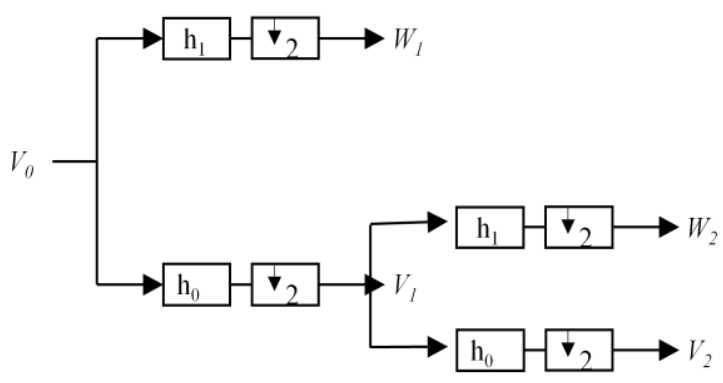

Fig.5 Dyadic wavelet decomposition 


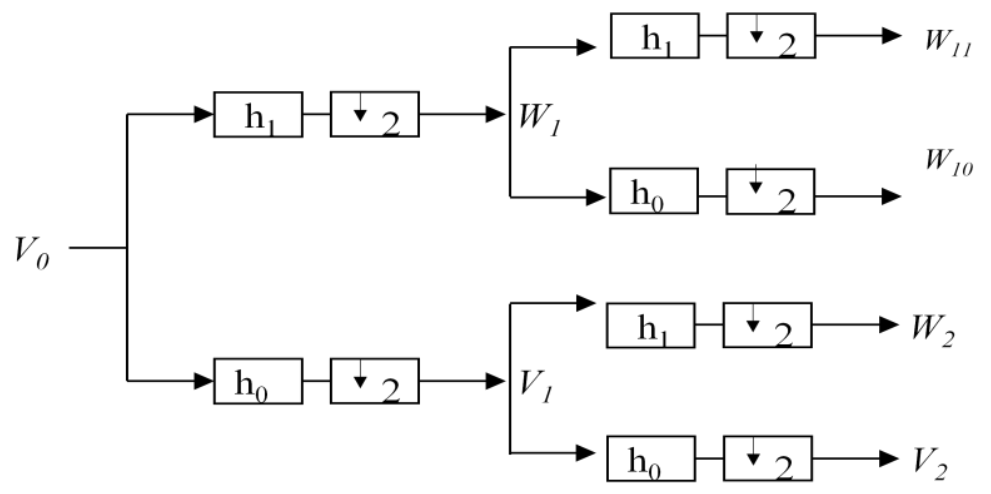

Fig.6 Wavelet packet decomposition

\section{DENOISING WITH WAVELET COEFFICIENTS THRESHOLDING}

The algorithm we have followed for denoising with wavelet coefficients thresholding is presented below.

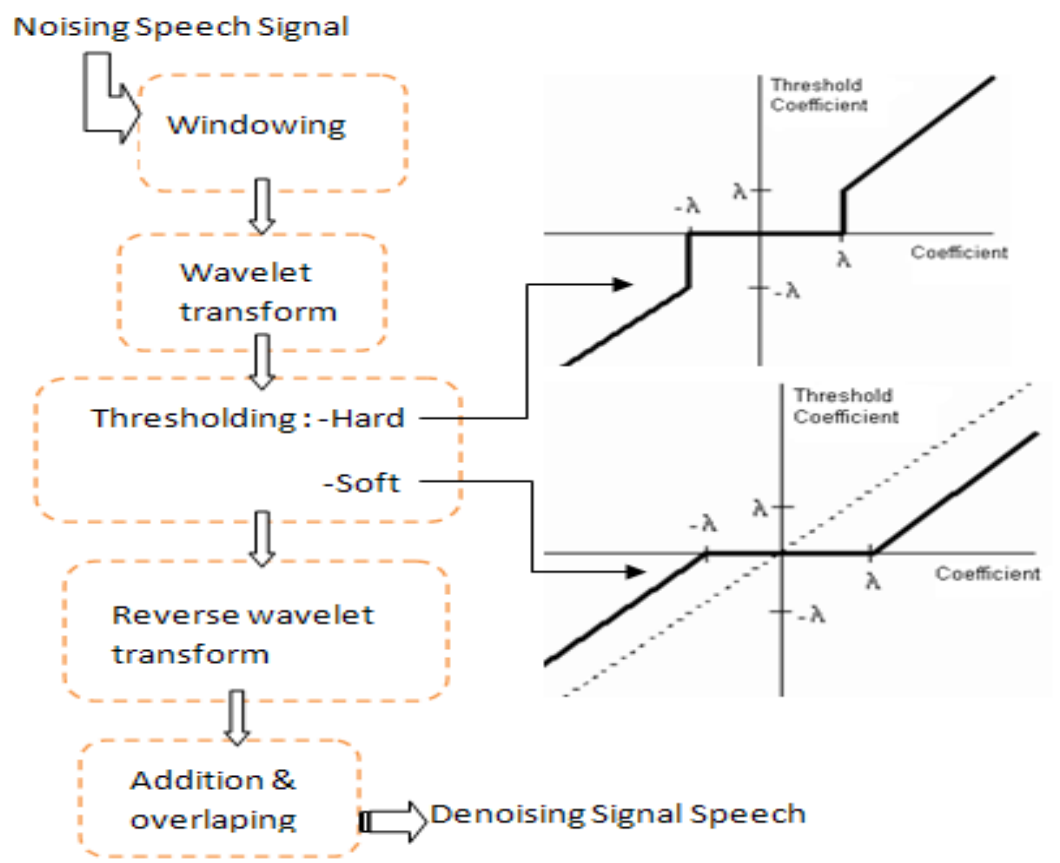

Fig.7 Wavelet analysis algorithm

\section{THRESHOLD DETERMINATION}

The threshold determination is performed using the following nonlinear function of contraction [19]:

$$
\begin{aligned}
& \operatorname{pour}|X|>T \\
& \operatorname{pour}|X| \prec T
\end{aligned}
$$

For each node N, the threshold is defined by the following formula:

$$
T_{j, k}=\hat{\sigma}_{j, k} \sqrt{2 \log (N)}
$$

The parameters $\mathrm{j}$ and $\mathrm{k}$ represent respectively the scale and the index of the band [20].

We take:

$$
\beta=0,9 \text { et } \mu=255
$$


- Daubechies wavelets of order 4.

- 3 decomposition levels.

\section{DENOISING ON TIMIT VOWELS}

\section{BASIS}

The gain in $\mathrm{dB}$ for different denoising techniques is given in the following table.

Table III. Comparative SNR over techniques of noise reduction

\begin{tabular}{|l|l|l|l|l|}
\hline \multirow{2}{*}{ Input SNR in dB } & \multicolumn{4}{|c|}{ Output SNR in dB } \\
\cline { 2 - 5 } & Spectral subtraction & Hard threshold & Soft threshold & Adapted threshold \\
\hline $10 \mathrm{~dB}$ & 13.20 & 10.10 & 10.30 & 11.60 \\
\hline $5 \mathrm{~dB}$ & 9.81 & 12.40 & 12.77 & 5.38 \\
\hline $0 \mathrm{~dB}$ & 6.74 & 10.65 & 11.41 & 1.72 \\
\hline
\end{tabular}

We note that the output gain increases with decreasing of

\section{RECOGNITION RESULTS BY SOM WITH NOISE REDUCTION}

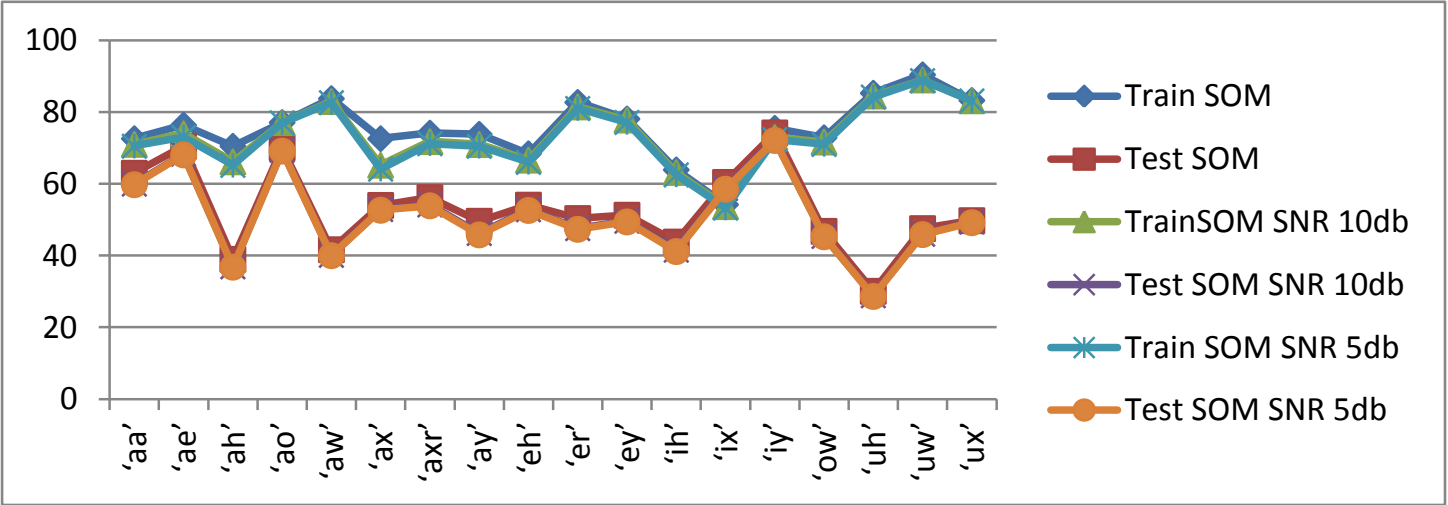

Fig.8 Vowels recognition rate of TIMIT database by the use of SOM joint to wavelet packet technique

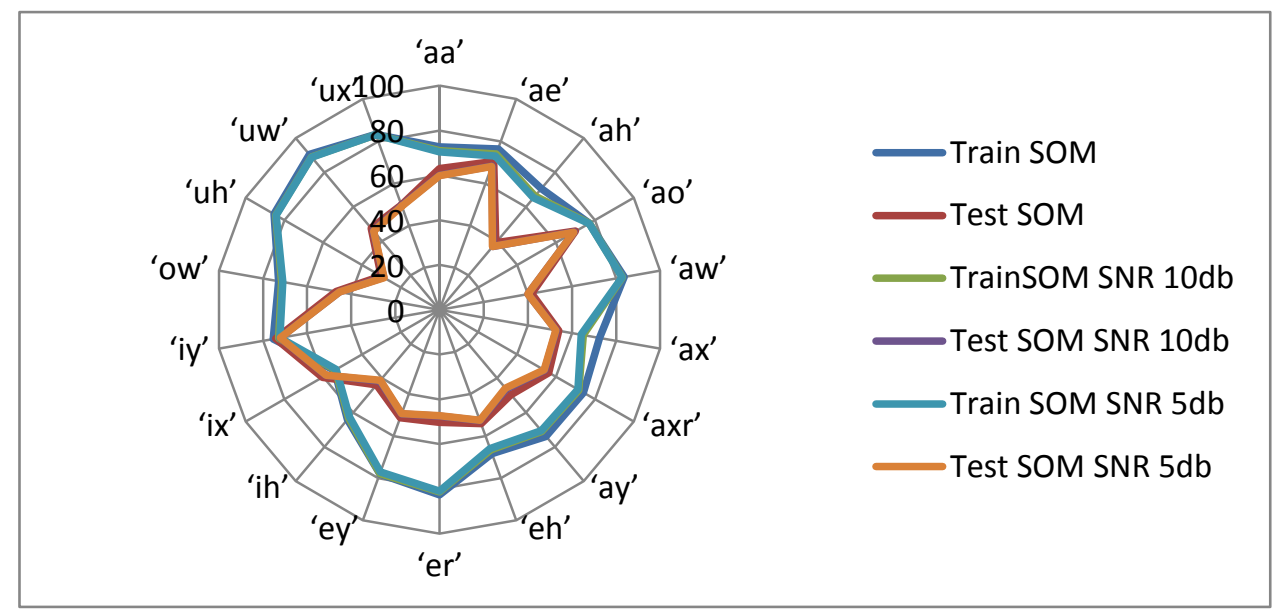

Fig.9 Comparative Vowels recognition rate of TIMIT database by the use of SOM joint to wavelet packet technique in radar form 


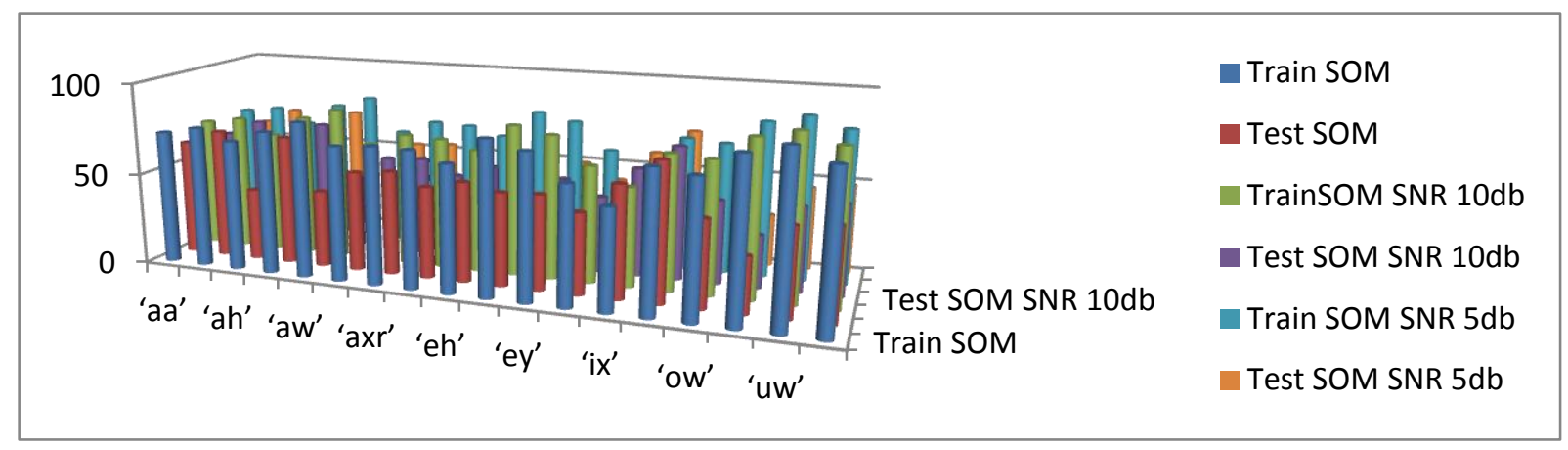

Fig.10 Comparative Vowels recognition rate of TIMIT database by the use of SOM joint to wavelet packet technique in histogram form

We note that there are areas of rapprochement between the generalization (test) rates and the self consistency (train) rates. The averages of generalization rate results based on vowels with and without noise reduction are listed in the table below. The technique of noise reduction is based on the method of adaptative wavelet packet thresholding.

Table IV. Overall rate of SOM generalization based on the vowels

\begin{tabular}{|l|l|l|l|l|}
\hline Original Signal & $\begin{array}{l}\text { SNR = 0dB without } \\
\text { noise reduction }\end{array}$ & $\begin{array}{c}\text { SNR = 0dB with } \\
\text { noise reduction }\end{array}$ & $\begin{array}{c}\text { SNR = 5dB with } \\
\text { noise reduction }\end{array}$ & $\begin{array}{c}\text { SNR = 10dB with } \\
\text { noise reduction }\end{array}$ \\
\hline $53 \%$ & $30 \%$ & $49 \%$ & $51 \%$ & $51.5 \%$ \\
\hline
\end{tabular}

In this effect, we note that the lost rates, by the noise presence, are earned via the technique of noise reduction by adaptative wavelet packet thresholding that we have designed.
Over the following figures, we present the results of vowels classification by SOM. The first one is used in adverse environment; the second one is used for a clean speech signal.

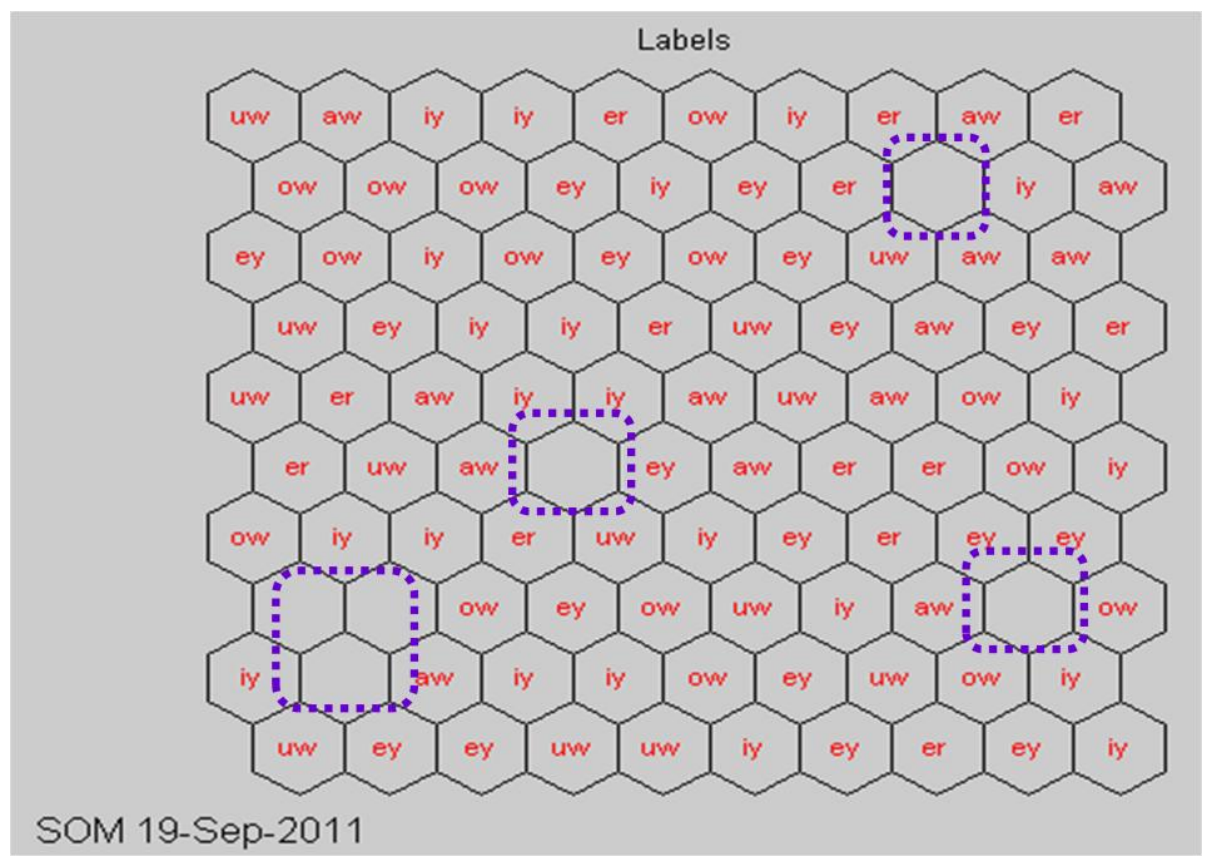

Fig.11 Representation of the SOM outputs for vowels input degraded by white Gaussian noise, SNR = 5 db, then denoised by wavelet packet at level 3 , after 20 learning iterations 


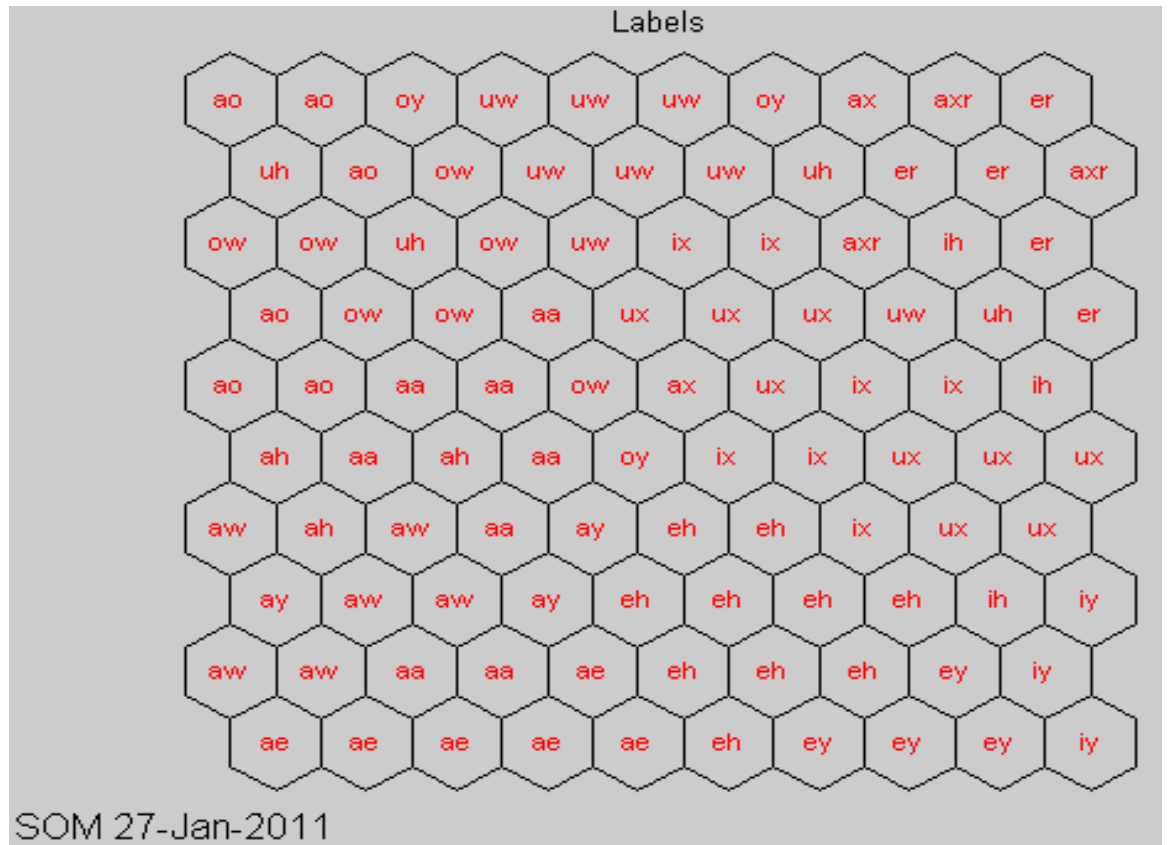

Fig.12 Representation of the SOM outputs for vowels input without noise, after 20 learning iterations

\section{CONCLUSION}

We have shown that the kohonen SOM map is a tool for unsupervised algorithm so it can be used in the recognition of a dictionary of a large data amounts.

The experimentation of this tool in the phonemes recognition in TIMIT database has validated its robustness. However, we showed that this robustness is weakened when using the SOM for recognizing a noisy signal. In order to improve the robustness of the SOM capacity in any adverse environment, we adopted a strategy of phonemes recognition by the joint use of SOM to the technique of noise reduction by adapted wavelet packet and we have obtained very encouraging results.

\section{Reference}

[1] Teuvo Kohonen,:' Self-organizing maps', Helsinki university of technology november 16, 2000.

[2] M. Berouti, R. Schwartz et J. Makhool : 'Enhancement of Speech Corrpted by acoustic Noise', Proceedings of the IEEE, pp 208-211, 1979.

[3] R.Boite, H Bourlard, T. Dutoit, J. Hancq et H. Leich : 'Traitement de la parole', Collection technique et Scientifique des Télécommunications, 1999.

[4] S.F Boll, : 'Suppression of Acoustic Noise in Speech Using Spectral subtraction', IEEE Transactions on Acoustics Speech and Signal Processing, Vol 27, pp 113120, April 1979.

[5] A. Bron : ' wavelet-based denoising of speech", Master Thesis, Technion Israel Institute of Technology, Haifa, 2000.

[6] B. Burke: ' Onde et ondelette la saga d'un outil mathématique', editions, Hubbard, 1996.

[7] R. R. Coifman et M.V. Wickerhauser : 'Entropy based Algorithm for best basis selection', IEEE transactions on Information theory, 1992.
[8] D. L. Donoho: "De-noising by Soft Thresholding", IEEE Transactions on Information Theory, vol 41, $\mathrm{n}^{\circ}$, May 1995.

[9] Y. Ephraïm and D. Malah : "Speech enhancement using a minimum meansquare error short-time spectral amplitude estimator", IEEE Trans. on Acoustics,Speech and Signal Processing, vol 32, n ${ }^{\circ}$ 6,pp 1109-1121, Dec 1984.

[10] Y. Ephraïm and D. Malah : "Speech enhancement using a minimum meansquare error log-spectral amplitude estimator", IEEE Trans. on Acoustics, Speech and Signal Processing, vol.33, n², pp. 443-445, Apr 1985.

[11] G. Ju, L. Lee: 'Speech enhancement and improved recognition accuracy by integrating wavelet transform and spectral subtraction algorithm', 8th European Conference on Speech Communication and Technology, Eurospeech2003.

[12] J.S. Lim et A.V Oppenheim: 'Enhancement and bandwidth compression of noisy speech', Proceedings of the IEEE, pp 1586-1604, 1979.

[13] S. Mallat: 'Une exploration des signaux en ondelettes', Editions de l'Ecole Polytechnique, 2000.

[14] T. F. Quatieri : 'Discrete time speech processing : Practice and Application', Prentice Hall, New Jersey, 2002.

[15] Stephan Bloehdorn and Sebastian Blohm: "A Self Organizing Map for Relation Extraction from Wikipedia using Structured Data Representations", Institute AIFB, University of Karlsruhe D-76128 Karlsruhe, Germany, 2006.

[16] F. Truchetet: 'Ondelettes pour le signal numérique'. Collection Traitement du Signal, Edition Hermes, 1998. 
[17] S. V. Vaseghi:"Advanced Signal Processing and Noise Reduction', John Whiley \& Sons edition, 1996.

[18] N. Whitmal, J . C. Rutledge et J. Cohen: "Reducing correlated noise in digital hearing aids", IEEE Eng. Med. Biol. Magazine, vol15, pp. 88-96, 1996.

[19] S. Chang, Y. Kwon, S. I. Yang and I. J. Kim : 'Speech Enhancement for non stationary noise Environment by adaptive Wavelet Packet', Proc. International Conference on Signal and Speech Processing, Vol 1, pp 561-564, 2002.

[20] M.S.Salhi and N. Ellouze : 'An Adaptative Thresholding SOM-Wavelet Packet Model to Improve the Phonemic Recognition Rate', Setit 2012_IEEE proceeding. 